\title{
sciendo
}

DOI: $10.2478 / f v-2020-0038$

FOLIA VETERINARIA, 64, 4: 55-62, 2020

\section{OCCURENCE AND ANTIMICROBIAL RESISTANT PATTERNS OF METHICILLIN RESISTANT STAPHYLOCOCCUS AUREUS (MRSA) AMONG PRACTICING VETERINARIANS IN KEBBI STATE, NIGERIA}

\author{
Gaddafi, M. S. ${ }^{1}$, Yakubu, Y. ${ }^{2}$, Garba, B. ${ }^{2}$ \\ Bello, M. B. ${ }^{3}$, Musawa, A. I. $^{2}$, Lawal, H. ${ }^{1}$ \\ ${ }^{1}$ Ministry of Animal Health, Husbandry and Fisheries, Kebbi State, \\ ${ }^{2}$ Department of Veterinary Public Health and Preventive Medicine, \\ ${ }^{3}$ Department of Veterinary Microbiology, Faculty of Veterinary Medicine Usmanu Danfodiyo University \\ Sokoto State \\ Nigeria
}

gaddafimohammedsani@yahoo.com

\section{ABSTRACT}

Methicillin-resistant Staphylococcus aureus (MRSA) is an emerging zoonotic pathogen incriminated in causing multiple disease conditions in humans and livestock. Studies have shown relationships between livestock rearing and increased MRSA colonization risk among farm workers, and also suggest that livestock may serves as reservoirs of the bacteria and could also infect humans via close contact and consumption of contaminated animal products. The aim of this study was to investigate if practicing veterinarians with significant livestock contacts are at risk for MRSA colonization. Therefore, a non-randomized survey was conducted to establish the presence of MRSA among veterinarians practicing in Kebbi State Nigeria, using both cultural characteristics and molecular detection of the resistant gene (mecA). Forty-one (41) nasal swabs were aseptically collected. The detection rate of MRSA in the veterinarians was $14.6 \%$. The study revealed a high occurrence rate of
MRSA among veterinarians in the study area. The relatively high prevalence recorded among veterinarians in this study could be attributed to the poor understanding of MRSA as a disease, its mode of transmission and its status in the country which have contributed immensely to the little/no awareness of MRSA among veterinarians and hence making it favourable for the bacteria (MRSA) to spread.

Key words: mecA; MRSA; Nigeria; practicing veterinarians; Staphylococcus aureus

\section{INTRODUCTION}

Staphylococcus aureus is a gram-positive opportunistic bacterium affecting both humans and other animals globally. The emergence of antimicrobial resistant strains of the bacteria has developed into a cause of concern as a serious public health problem [33]. Several species of staphylococci 
have been recognized and some of them have been isolated as commensals in humans with enormous pathogenic potentials [37]. On mannitol salt agar, S. aureus grows as golden yellow colonies [30]. S. aureus is a facultative anaerobe, growing well under aerobic conditions within 24 hours [12]. It is found in people and frequently colonizes the nares, the armpit, the perineum, skin folds and the vagina without giving rise to disease [23]. It is a major opportunistic pathogen that is incriminated in a range of chronic and acute infections of humans and other animals worldwide [36]. A fundamental biological property of Staphylococcus aureus is its ability to colonize both healthy and hospitalized people asymptomatically; most often involving the anterior nares [11]. Staphylococcus aureus is a highly pathogenic organism capable of causing multiple diseases in humans. Its success as a commensal organism and pathogen is because of its versatility and rapid adaptability [31]. Mobile genetic elements (MGEs) as a means for the transfer of genetic information between and within bacterial species play a critical role in its adaptation process [24].

As a "superbug", S. aureus has high morbidity and mortality rates due to its ability to mutate, therefore endowing it with considerate levels of resistance to different classes of antibiotics recommended for their treatment [15]. Antimicrobial resistance to $S$. aureus is a major public health threat, compounded with the emergence of vancomycin and daptomycin resistant strains which are the last line of antimicrobials [19]. There has been a global increase of MRSA over the past few decades and livestock associated methicillin resistant Staphylococcus aureus (LAMRSA) has remained endemic in certain groups of workers with direct contact to live animals [41]. Methicillin-resistant Staphylococcus aureus (MRSA) has been reported in different species of animals and their products [44]. Animals can serve as reservoirs and also as a source for the emergence of new clones of methicillin-resistant Staphylococcus aureus (MRSA) in humans [16]. Recently, a new sequence type belonging to the clonal complex 398 has emerged in pigs and calves worldwide causing invasive disease in humans [38]. And since its emergence in the early 2000s, the new strain of MRSA has increasingly become the cause of human infections most especially in those with livestock exposure [35].

The presence of LA-MRSA in farm workers is a major threat to public health and the health care system [45]. The prevalence of MRSA in humans has been shown to be strongly associated with its prevalence in animals and the intensity of contact with animals positive for methicillin-resistant Staphylococcus aureus [17]. MRSA can be transmitted between humans and their pets, although the route of transmission and risk factors for transmission are not well understood [45]. B r o n w y n et al. [8] described a case of human-to-dog transmission of MRSA. Several researchers postulate that MRSA infection and colonization in household pets strongly suggests that pets tend to be infected or colonized with MRSA strain types from community human populations $[23,45]$. Also, researchers believed that MRSA in pets are closely linked to MRSA infection in humans and that infected or colonized humans may often be the source of MRSA in household animals [23]. N w o ri e et al. [29] reported a prevalence of $23 \%$ of $S$. aureus amongst meat sellers in Abakaliki metropolis, Ebonyi State, Nigeria. Nonetheless, the presence of methicillin resistant Staphylococcus aureus has been documented in Nigeria among farm animals and farm workers [30, 42].

Despite the MRSA colonization rate in animals and their potential to transmit the infection to veterinarians, there has not been any molecular detection of MRSA among practicing veterinarians in Kebbi State, Nigeria, nor a documentation of its involvement in staphylococcal infections in livestock in the State. Therefore, this study was aimed at investigating the presence of MRSA among veterinarians in Kebbi State, Nigeria.

\section{MATERIALS AND METHODS}

\section{Study area}

The sampling locations for this study were the Zonal Veterinary Clinics in Kebbi State, Nigeria. The state is located in North-Western Nigeria, which lies between $12.45^{\circ} \mathrm{N}$ latitude and $4.2^{\circ} \mathrm{E}$ longitude.

\section{Sample collection}

A cross sectional study approach using non-randomized sampling was adopted. Considering the zoonotic potential of the disease, gloves were used during the collection of the samples. After seeking the veterinarian's consent, a nasal swab was taken using a sterile swab and labelled appropriately. Forty-one (41) veterinarians were sampled across the state. The study was conducted between April and June 2019. 


\section{Ethical clearance}

All the sampling procedures were done in accordance with the Animal Research Ethics Committee of the Faculty of Veterinary Medicine, Usmanu Danfodiyo University Sokoto (UDUS/FAREC/03/2019).

\section{Bacteriological culture and isolation}

Mannitol salt agar (OXOID) was prepared according to the manufacturer's instructions. The prepared agar was then poured into petri dishes and the prepared plates were then incubated at $37^{\circ} \mathrm{C}$ for 24 hours to ascertain the sterility of the plates before inoculation of the samples (sterility test). The swab stick containing the sample was used to inoculate the plates by creating a primary well and then a sterile wire loop was used for streaking in order to obtain discrete colonies. The streaked plates were incubated at $37^{\circ} \mathrm{C}$ for 24 hours. Presumptive colonies of Staphylococcus aureus species appearing as small, smooth, golden shiny convex colonies with golden yellow zones on Mannitol salt agar were picked and inoculated onto nutrient agar slants, and further incubated at $37^{\circ} \mathrm{C}$ for another 24 hours.

\section{Biochemical analysis}

All of the colonies grown on nutrient agar medium were subjected to the following conventional biochemical tests such as catalase, and coagulase tests for the identification of Staphylococcus species.

The catalase test was carried out as described by $\mathrm{O}$ ch e i and Ko h lh at k a r [30], while the slide agglutination test to determine coagulase positive isolates was carried out as described by $\mathrm{C}$ h e e s b r o u g h [12].

\section{Phenotypic characterization of MRSA}

Oxacillin resistance Staphylococcus aureus broth (ORS$\mathrm{AB}$; Oxoid) was prepared according to the manufacturer's instructions. Presumptively, S. aureus colonies based on biochemical test were inoculated on prepared ORSAB plates. The presumptive MRSA (oxacillin resistant strains) colonies appeared as bright blue on the media.

\section{Antimicrobial susceptibility test}

The Kirby-Bauer disk diffusion method was used to determine the antimicrobial susceptibility of the isolates on Mueller Hinton agar using a panel of antimicrobials including; erythromycin, oxytetracycline, neomycin, penicillin, sulphonamides, gentamycin and vancomycin. The procedure and interpretations were based upon the recommendations of the Clinical and Laboratory Standards Institute [14]. Resistance or susceptibility of the isolates against the drugs tested were determined based on zones of inhibition measured to the nearest millimetre using a calliper. The sizes of the zones of inhibition were interpreted by comparing with the breakpoints as described by Clinical and Laboratory Standard Institute [14].

\section{GENOTYPIC CHARACTERIZATION OF MRSA}

\section{Genomic DNA extraction}

Genomic DNA was extracted using the traditional boiling method as described by $\mathrm{C}$ h e $\mathrm{n}$ et al., [13] with slight modifications. A loop-full suspension of overnight grown cultures on nutrient agar plate was transferred into a $1.5 \mu \mathrm{l}$ microcentrifuge tube containing $200 \mu \mathrm{l}$ of sterile distilled water. The suspension was first incubated at room temperature for $5 \mathrm{~min}$, and then heated in a dry water bath at $96^{\circ} \mathrm{C}$ for $10 \mathrm{~min}$. This was then followed by centrifugation at $12,000 \mathrm{~g}$ for $5 \mathrm{~min}$., and the supernatant was then collected in a new $1.5 \mu$ l tube using a micropipette and then used as the DNA template.

\section{MecA gene amplification}

The DNA of all of the positive ORSAB S. aureus isolates were subjected to amplification by polymerase chain reaction (PCR) for the presence of the $163 \mathrm{bp}$ fragment of the mecA gene using the following primers; mecA1 5'AAAATCGATGGTAAAGTTGGC-3'(forward), mecA2 5'AGTTCTGCAGTACCGGATTTGC-3'(reverse) [26]. The PCR was performed using the Qiagen Master mix kit in a $25 \mu \mathrm{l}$ reaction mixture containing $3 \mu \mathrm{l}$ of nuclease free water, $5 \mu \mathrm{l}$ of the DNA template, $1 \mu \mathrm{l}$ of each forward and reverse primer, $2.5 \mu \mathrm{l}$ of coral load (loading dye, Qiagen) and $12.5 \mu \mathrm{l}$ of the master mix (Qiagen). The PCR amplification protocol comprises of 35 cycles of amplification with an initial denaturation of $94^{\circ} \mathrm{C}$ for $5 \mathrm{~min}$, denaturation at $94^{\circ} \mathrm{C}$ for $1 \mathrm{~min}$, annealing at $57^{\circ} \mathrm{C}$ for $1 \mathrm{~min}$, extension at $72{ }^{\circ} \mathrm{C}$ for $1 \mathrm{~min}$ and then final extension at $72{ }^{\circ} \mathrm{C}$ for 7 min (GeneAmp PCR System 9700). The PCR products were visualized after electrophoresis for $45 \mathrm{~min}$ at 90 volts in a $1 \%$ agarose gel. The bands were then viewed under a UV trans-illuminator. 
Table 1. Antibiotic susceptibility profile of MRSA isolates $(n=10)$ from veterinarians in Kebbi State, Nigeria

\begin{tabular}{lcccc}
\hline Antibiotics & $\begin{array}{c}\text { Breakpoints } \\
{[\mathrm{mm}]}\end{array}$ & Resistant & $\begin{array}{c}\text { Inter. } \\
\text { mediate }\end{array}$ & Susceptible \\
\hline Erythromycin & $14-22$ & 5 & 5 & - \\
Oxytetracycline & $14-15$ & 5 & 5 & - \\
Neomycin & $13-14$ & 6 & 4 & - \\
Penicillin & $17-18$ & 10 & - & - \\
Sulphonamides & $13-16$ & 6 & 3 & 1 \\
Gentamycin & $13-17$ & 7 & 3 & - \\
Vancomycin & $15-19$ & - & - & 10 \\
\hline
\end{tabular}

Note: The breakpoints (in millimetres) are based on the CLSI standard (2008)

Table 2. Antibiotic resistant pattern for MRSA isolates from veterinarians in Kebbi State, Nigeria.

\begin{tabular}{lc}
\hline Antibiotic resistance pattern & Number of isolates \\
\hline Ery-Oxy-Neo-Pen-Sul-Gen & 4 \\
Ery-Oxy-Neo-Pen-Gen & 1 \\
Ery-Pen-Sul-Gen & 1 \\
Ery-Pen-Gen & 1 \\
Oxy-Neo-Pen-Sul-Gen & 1 \\
Oxy-Neo-Pen-Sul & 1 \\
Neo-Pen-Sul-Gen & 1 \\
\hline
\end{tabular}

Ery-Erythomycin; Oxy-Oxytetracyclin; Neo-Neomycin; Pen-Penicillin; Sul-Sulfonamide; Gen—Gentamycin

\section{Data analysis}

The results obtained in this study which were presented in tables and percentages, were computed using Microsoft Excel programme version 2010.

\section{RESULTS}

Among the 41 samples cultured, 17 (41.5\%) had colonial growth and biochemical characteristics synonymous to Staphylococcus sp. However, only ten (10) out of the 41 (24.4\%) samples were MRSA positive based on the phenotypic characterization and bright blue pigmentation on the ORSAB media.

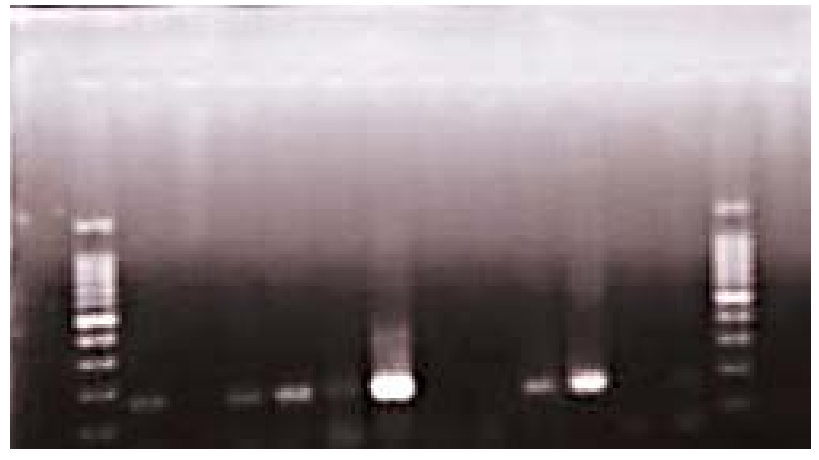

Fig. 1. Agarose gel electrophoresis image showing amplification of mecA gene (163bp). Lane $\mathrm{L}-100$ bp ladder; lane $\mathrm{P}$-positive control; lanes $\mathrm{N}$-negative control; lanes $1,2,3,4,7,8$-positive samples; lanes 5, 6, 9, 10-negative samples

The results for the antimicrobial susceptibility tests revealed that the ORSAB positive isolates exhibited varying level of resistance against the antimicrobials tested, with five (50\%) showing resistance to erythromycin and oxytetracycline, six $(60 \%)$ of the isolates were resistant to neomycin and sulfonamides, and 7 (70\%) of them were resistant to gentamycin. Additionally, all of the MRSA isolates were seen to be resistant to penicillin, while all of the isolates were susceptible to vancomycin on the other hand (Table 1). However, four of the isolates were found to show multidrug resistance (MDR) with Ery-Oxy-Neo-Pen-SulGen being the most common pattern (Table 2).

Similarly, PCR amplification of all the ten (10) positive samples on ORSAB for the detection of mecA gene showed that $60 \%(6 / 10)$ of the isolates possess the mecA gene (Fig. 1). Hence, the overall molecular detection rate of MRSA among the veterinarians studied is $14.6 \%(6 / 41)$.

\section{DISCUSSION}

MRSA has been reported in different livestock including cattle, poultry and pigs $[6,21]$. Several studies have indicated MRSA transmission from livestock to farm workers [20], but the exact extent of transmission between livestock and farm workers alongside with its clinical significance remain unclear; in the same vein whether individuals in walks of life other than animal rearing are at risk of becoming MRSA carriers remains unknown. The overall detection rate of MRSA among veterinarians in this study was $14.6 \%$. The prevalence recorded in this study were similar to the 
prevalence reported by $\mathrm{M}$ idd le t o $\mathrm{n}$ et al. [27] who recorded a prevalence of $14 \%$ in veterinarians at a veterinary teaching hospital in the USA. Also, L o e ffl e r et al. [23] reported a prevalence of $18 \%$ among the veterinary staff of a small animal hospital in the UK. W e e s e [45] reported a prevalence of $13 \%$ among veterinary personnel that came in contact with animals positive with MRSA in Canada. The similarities noticed in the prevalence above could be attributed to the same technique of detection and the sample size used in our study. S e g u i n et al. [39] reported a nasal carriage of MRSA in three (3) of five (5) veterinary staff during an outbreak of MRSA in an equine hospital in the USA. Our prevalence was much higher than that reported by A budu et al. [1] and Maudsley et al. [25] that reported $1.5 \%$ and $0.8 \%$ respectively in a community survey conducted among the veterinary staff in the UK. This also can be partly attributed to the fact that the endemicity of MRSA was higher in Nigeria than the UK and also the irrational use of antibiotics (beta-lactam) in animals in Nigeria was higher than in the UK which can lead to increased MRSA spread and hence may explain the higher prevalences recorded. W ulf et al. [47] reported a prevalence of $3.9 \%$ among veterinarians. Also, A kil u et al. [3] showed a prevalence of $7 \%$ among veterinary staff at a veterinary hospital in Malaysia. The relatively high prevalence recorded among veterinary staff in our study could be attributed to the poor understanding of MRSA as a disease, its mode of transmission and its economic impact to healthcare in the country which have also contributed greatly to lack of awareness of MRSA among veterinarians and thus making it favourable for the bacteria (MRSA) to spread. Also, in Nigeria, antimicrobial agents are still readily available to people in local drug stores over the counter, a practice which has led to misuse of antibiotics with the associated high prevalence of antibiotic resistance among Staphylococcus [27]. Nuttall et al. [28] suggested that MRSA could be an occupational hazard to individuals in the animal health profession. Numerous studies have suggested that animals may serve as a reservoir for human infection with MRSA [20]. H a n s e $1 \mathrm{~m}$ a $\mathrm{n}$ et al. [18], W u lf et al. [47], W illi a m s et al. [46] and A nde r s o n et al. [5] reported that veterinarians have much higher MRSA colonization rates when compared with the general population. I s $\mathrm{h}$ i h a r a et al. [20] suggested that in a veterinary hospital, the veterinary staff might be a primary source of MRSA infection for the animals.
Slight differences between phenotypic and genotypic detection techniques were also observed in our study. Notwithstanding, all isolates that appeared as bright blue on ORSAB were presumed to be MRSA because ORSAB has been commonly used for the detection MRSA with $99 \%$ sensitivity in the detection of MRSA as reported by $\mathrm{B}$ e c k e r et al., [7]. The gene responsible for the resistance (mecA) was detected in $60 \%(6 / 10)$ of the isolates that were ORSAB positive in our study. Even though the presence of mecA has been recognized as the gold standard for MRSA detection as reported by Aliy u et al., [3], studies have shown that there exist mechanisms that are non-mec-dependent and they may contribute towards methicillin resistance in staphylococci strains [8, 9]. A li y u et al., [4], Us man and Mustapha [43], Shamila-S y u ha$\mathrm{d}$ a et al. [40], and $\mathrm{Pu}$ et al. [36] all reported differences in phenotypic and genotypic detection of MRSA in S. aureus. So, these discrepancies noticed between the phenotypic and genotypic methods of detection could be attributed to the non-mecA-dependent methicillin resistance and heterogeneous expression of methicillin resistance in S. aureus. Also, the discrepancy between the results obtained in our study and those of the aforementioned researchers above, can be attributed to the postulation of L e e et al. [22], who opined that phenotypic resistance of $S$. aureus to methicillin could vary depending on cellular growth conditions. Phenotypic expression of resistance to methicillin in MRSA varies, and each strain has a characteristic profile of the proportion of bacterial cells that grow at specific concentrations of methicillin according to $\mathrm{Pl}$ a t a et al. [34]. It has also been observed by A d e s i d a et al. [2] that PCR detection of mecA gene does not always give indisputable results. Some MRSA strains have been found to be mecA-negative in PCR, but resistant to methicillin. Furthermore, some MRSA strains have also been reported to be mecA positive but susceptible to methicillin (Olo nit ol a et al. [32]). The absence of mecA in MRSA strains could also be an indication of the potential presence of "modified S. aureus" (MODSA). MODSA possess modified penicillin-binding proteins (PBPs) which was a different classical mechanism of resistance to methicillin in MRSA according to $\mathrm{B} \mathrm{h}$ u t i a et al. [10]. In this study, a negative correlation was found between the phenotypic resistance to methicillin and PCR results for the detection of mecA gene that code for resistance to methicillin. 


\section{CONCLUSIONS}

The overall prevalence of MRSA in veterinarians in our study was $14.6 \%$. This suggests that animal health workers could be at a higher risk of MRSA asymptomatic colonization and this could be attributed to occupational exposure with animals, therefore we concluded that, veterinarians caring for livestock have a high risk of being colonized by MRSA. The public health implication of our study was that veterinarians with professional contact with livestock are frequently colonized with livestock-acquired (LA) MRSA. As such, these individuals are presumably the source for LA-MRSA transmission to household members and other parts of the human population. The infection of these group of professionals leads to the introduction of LA-MRSA into the hospital and other healthcare facilities. Further studies are needed to determine the exact source of MRSA in both animal care providers and the livestock. More so, in Nigeria, the indiscriminate use of antibiotics in livestock production could have also contributed immensely to the patterns of antibiotic resistance recorded in our study although the sample size in our study was not large enough to make a generalization of the situation, we strongly believe that it provided evidence of the occurrence of MRSA in veterinarians in Kebbi State Nigeria and hence can serve as a baseline data for further investigations.

\section{ACKNOWLEDGEMENTS}

The authors wish to thank the entire staff of the veterinary services Department, and the Zonal veterinary clinics, Ministry of Animal Health, Husbandry and Fisheries, Kebbi State for their cooperation during the period of sampling.

\section{REFERENCES}

1. Abudu, L., Blair, I., Fraise, A., Cheng, K. K., 2001: Methicillin-resistant Staphylococcus aureus (MRSA): a community-based prevalence survey. Epidemiol. Inf., 126, 351-356. DOI: $10.1017 / \mathrm{s} 0950268801005416$.

2. Adesida, S. A., Bocleas, H., Babajide, B., Kehinde, A., Saijders, S., van Leeuwen, W., et al., 2005: Major epidemic clones of Staphylococcus aureus in Nigeria. Microb. Drug Resist., 11, 2, 115-121. DOI: 10.1089/mdr.2005.11.115.
3. Aklilu, E., Zunita, Z., Hassana, L., Chenga, H. C., 2013: Molecular epidemiology of methicillin-resistant Staphylococcus aureus (MRSA) among veterinary students and personnel at a veterinary hospital in Malaysia. J. Vet. Microbiol., 164, 352-358. DOI: 10.1016/j.vetmic.2013.02.030.

4. Aliyu, Y., Abdullahi, I. O., Whong, C. Z., Olalekan B. O., Reuben, R. C., 2020: Occurrence and antibiotic susceptibility of methicillin resistant Staphylococcus aureus in fresh milk and milk products in Nasarawa State, North-Central Nigeria. J. Microbiol. Antimicrob., 12, 1, 32-41. DOI: 10.5897/JMA 2020.0424 .

5. Anderson, M. E. C., Lefebvre, S. L., Weese, J. S., 2008: Evaluation of prevalence and risk factors for methicillin-resistant Staphylococcus aureus colonization in veterinary personnel attending an international equine veterinary conference. J. Vet. Microbiol., 129, 410-417. DOI: 10.1016/j.vetmic.2007.11.031.

6. Armand-Lefevre, L., Ruimy, R., Andremont, A., 2005: Clonal comparison of Staphylococcus aureus isolates from healthy pig farmers, human controls, and pigs. Emerg. Inf. Dis., 11, 711-714. DOI: 10.3201./eid1105.040866.

7. Becker, A., Forster, D. H., Kniehl, E., 2002: Oxacillin resistance screening agar base for detection of methicillin-resistant Staphylococcus aureus. J. Clin. Microbiol., 40, 4400-4401. DOI: $10.1128 / \mathrm{jcm} .40 .11 .4400-4401.2002$.

8. Berger-Bachi, B., 1995: Factors affecting methicillin resistance in Staphylococcus aureus. Int. J. Antimicrob. Agents, 6, 13-21. DOI: 10.1016/0924-8579(95)00021-Y.

9. Berger-Bachi, B., Tschierske, M., 1998: Role of Fem factors in methicillin resistance. J. Drug Resist. Updates, 1, 325-335. DOI: 10.1016/s1368-7646(98)80048-4.

10. Bhutia, K. O., Singh, T. S., Biswas, S., Adhikari, L. 2012: Evaluation of phenotypic with genotypic methods for species identification and detection of methicillin resistant in Staphylococcus aureus. Int. J. Appl. Basic Med. Res., 2, 2, 84-91. DOI: 10.4103/2229-516X.106348.

11. Bien, J., Sokolova, O., Bozko, P., 2011: Characterization of virulence factors of Staphylococcus aureus: Novel function of known virulence factors that are implicated in activation of airway epithelial pro-inflammatory response. J. Pathog., Epub., 2011, 1-13. DOI: 10.4061/2011/601905.

12. Cheesbrough, M., 2010: District Laboratory Practice in Tropical Countries Part II. Cambridge University Press, Cambridge, UK, 65, 38-39. DOI: 10.1017/cbo9780811581304.

13. Chen, L., Mediavilla, J. R., Oliveira, D. C., Willey, B. M., de Lencastre, H., et al., 2009: Multiplex real-time PCR for rap- 
id staphylococcal cassette chromosome mecA typing. J. Clin. Microbiol., 47, 3692-3706. DOI: 10.1128/jmc.00766-09.

14. Clinical and Laboratory Standards Institute, 2008: Performance Standards for Antimicrobial Susceptibility Testing; Eighteenth Informational Supplement, M100-S18, 28, 1.

15. Davies, J., Davies, D., 2010: Origins and evolution of antibiotic resistance. Microbiology. Molecular Biol. Rev., 74, 3, 417-433. DOI: 10.1128/mmbr.00016-10.

16. García-Álvarez, L., Holden, M. T. G., Lindsay, H., Webb, C. R., Brown, D. F. J., Curran, M. D., et al., 2011: Methicillin-resistant Staphylococcus aureus with a novel mecA homologue in human and bovine populations in the UK and Denmark: A descriptive study. Lancet Inf. Dis., 11, 8, 595-603. DOI: 10.1016/s1473-3099(11)70126-8.

17. Graveland, H., Wagenaar, J. A., Heesterbeek, H., Mevius, D., van Duijkeren, E., Heederik, D., 2010: Methicillin resistant Staphylococcus aureus ST398 in veal calf farming: Human MRSA carriage related with animal antimicrobial usage and farm hygiene. PLOS ONE, 5, 6, 1-6. DOI: 10.1371/journal. pone.0010990.

18. Hanselman, B. A., Kruth, S. A., Rousseau, J., Low, D. E., Willey, B. M., McGeer, A., Weese, J. S. 2006: Methicillin-resistant Staphylococcus aureus colonization in veterinary personnel. Emerg. Inf. Dis., 12, 1933-1938. DOI: 10.3201/eid 1212.060231

19. Howden, B. P., McEvoy, C. R. E., Allen, D. L., Chua, L., Gao, W., Harrison, P. F., et al., 2011: Evolution of multidrug resistance during Staphylococcus aureus infection involves mutation of the essential two component regulator WalKR. PLOS Pathol., 7, 11, 1002359. DOI: 10.1371/journal. ppat.1002359.

20. Ishihara, K., Saito, M., Shimokubo, N., Muramatsu, Y., Maetani, S., Tamura, Y., 2015: Methicillin-resistant Staphylococcus aureus carriage among veterinary staff and dogs in private veterinary clinics in Hokkaido, Japan. J. Microbiol. Immunol., 58, 149-154. DOI: 10.1111/1348-0421.12128.

21. Jensen, S. O., Lyon, B. R., 2009: Genetics of antimicrobial resistance in Staphylococcus aureus. Future of Microbiol., 4, 5, 565-582. DOI: 10.2217/fmb.09.30.

22. Lee, J. H., Jeong, J. M., Park, Y. H., Choi, S. S., Kim, Y. H., Chae, J. S., 2004: Evaluation of the methicillin-resistant Staphylococcus aureus of animal origin. J. Clin. Microbiol., 42, 6, 2780-2782. DOI: 10.1128/jcm.42.6.2780-2782.2004.

23. Loeffler, A., Pfeiffer, D. U., Lloyd, D. H., Smith, H., Lindsay, J. A., 2010: Methicillin resistant Staphylococcus aureus carriage in UK veterinary staff and owners of infected pets: new risk group. J. Hospit. Inf., 74, 3, 282-288. DOI: 10.1016/j. jhin.2009.09.020.

24. Lupo, A., Coyne, S., Berendonk, T. U., 2012: Origin and evolution of antibiotic resistance: The common mechanisms of emergence and spread in water bodies. Front. Microbiol., 3, 18, 1-13. DOI: 10.3389/fmicb.2012.00018.

25. Maudsley, J., Stone, S. P., Kibbler, C. C., Iliffe, S. R., Conaty, S. J., Cookson, B. D., et al., 2004: The community prevalence of methicillin-resistant Staphylococcus aureus (MRSA) in older people living in their own homes: implications for treatment, screening and surveillance in the UK. J. Hosp. Inf., 57, 258-262. DOI: 10.1016/j.jhin.2004.03.023.

26. Mehrotra, M., Wang, G., Johnson, W. M., 2000: Multiplex PCR for detection of genes for Staphylococcus aureus enterotoxins, exfoliative toxins, toxic shock syndrome toxin 1 and methicillin resistance. J. Clin. Microbiol., 38, 3, 1032-1035. DOI: $10.1128 / \mathrm{jcm} .38 .3 .1032-1035.2000$.

27. Middleton, J. R., Fales, W. H., Luby, C. D., Oask, J. L., Sanchez, S., Kinyon, J. M., et al., 2005: Surveillance of Staphylococcus aureus in veterinary teaching hospitals. J. Clin. Microbiol., 43, 2916-2919. DOI: 10.1128/jcm.43.6.2919.2005.

28. Nuttall, T., Williams, N., Saunders, R., Dawson, S., 2008: Methicillin resistant Staphylococci in companion animals. Eur. J. Comp. Anim. Pract., 18, 280-287.

29. Nworie, A., Azi, S. O., Ibiam, G. A., Egwu, I. H., Odoh, I., Okereke, E. C., et al., 2013: Nasal carriage of methicillin resistant Staphylococcus aureus amongst meat sellers in Abakaliki Metropolis, Ebonyi State, Nigeria. Microbiol. Res. Int., 1, 3, 48-53.

30. Ochei, J., Kolhatkar, A., 2000: Medical Laboratory Science-Theory and Practice. Tata McGraw-Hill Publishing Company Ltd, New Delhi, 648—657.

31. Olayinka, B. O., Bala, H. K., Ehinmidu, J. O., Onaolapo, J. A., 2010: Multidrug resistant Staphylococcus aureus isolates from poultry farms in Zaria, Nigeria. In 14th International Symposium on Staphylococci and Staphylococcal Infections, Bath, UK, ISSSI2010.

32. Olonitola, O. S., Olayinka, B. O., Onaolapo, J. A., 2007: Absence of mecA gene in MRSA isolates from non-hospital source in Zaria, Nigeria. Int. J. Nat. Appl. Sci., 13, 2, 160-164.

33. Otto, M., 2010: Basis of virulence in community-associated methicillin-resistant Staphylococcus aureus. Ann. Rev. Microbiol., 64,143-162.

34. Plata, K. B., Riosa, S., Singh, C. R., Rosato, R. R., Rosato, A. E. 2013: Targeting of PBP1 by beta-lactams determines recA/SOS response activation in heterogeneous MRSA clin- 
ical strains. PLOS ONE, 8, 4, 61083. DOI: 10.1371/journal. pone. 0061083.

35. Price, L. B., Stegger, M., Hasman, H., Aziz, M., Larsen, J., Andersen, P. S., et al., 2012: Staphylococcus aureus CC398: Host adaptation and emergence of methicillin resistance in livestock. Mol. Biol., 3, 1, 1-6. DOI: 10.1128/mBio.00305-11.

36. Pu, W., Su, Y., Li, C., Yang, Z., Deng, H., et al., 2014: High incidence of oxacillin-susceptible mecA-positive Staphylococcus aureus (OS-MRSA) associated with bovine mastitis in China. PLOS ONE, 9, 2, e88134. DOI: 10.1371/journal.pone. 0088134.

37. Rodriguez-Noriega, E., Seas, C., Guzma'n-Blanco, M., Mejia, C., Alvarez, C., Bavestrello, L., et al., 2010: Evolution of methicillin-resistant Staphylococcus aureus clones in Latin America. Int. J. Inf. Dis., 14, 7, 560-566. DOI: 10.1016/j.ijid. 2009.08.018.

38. Schijffelen, M. J., Boel, C. H. E., van Strijp, J. A. G., Fluit, A. C., 2010: Whole genome analysis of a livestock-associated methicillin-resistant Staphylococcus aureus ST398 isolate from a case of human endocarditis. BMC Genomics, 11, 376, 1-10. DOI: 10.1186/1471-2164-11-376.

39. Seguin, J. C., Walker, R. D., Caron, J. P., Kloos, W. E., Geworge, C. G., Hollis, R. J., et al., 1999: Methicillin-resistant Staphylococcus aureus. Outbreak in veterinary teaching hospital: potential human to animal transmission. J. Clin. Microbiol., 37, 1459-1463. DOI: 10.1128/jcm.37.5.1459-1463. 1999.

40. Shamila-Syuhada, A. K., Rusul, G., Wan-Nadiah, W. A., Chuah, L., 2016: Prevalence and antibiotic resistance of Staphylococcus aureus isolates isolated from raw milk obtained from small-scale dairy farms in Penang, Malaysia. Pak. Vet. J., 36, 1, 98-102.
41. Stefani, S., Chung, D. R., Lindsay, J. A., Friedrich, A. W., Kearns, A. M., Westh, H., et al., 2012: Methicillin-resistant Staphylococcus aureus (MRSA): Global epidemiology and harmonisation of typing methods. Int. J. Antimicrob. Agents, 39, 273-282. DOI: 10.1016/j.ijantimicag.2011.09.030.

42. Umaru, G. A., Kabir, J., Adamu, N., Umar, Y. A., 2011: A review of emerging methicillin-resistant Staphylococcus aureus (MRSA): A growing threat to veterinarians. Nigerian Vet. J., 32, 3, 174-186.

43. Usman, R. Z., Mustapha, B. M., 2016: Isolation and identification of methicillin- resistant Staphylococcus aureus (MRSA) from traditionally fermented milk "nono" and yoghurt in Kaduna metropolis, Nigeria. Food Sci. Qual. Manag., 55, 45-50. ISSN: 2225-0557.

44. Waters, A. E., Contente-Cuomo, T., Buchhagen, J., Liu, C. M., Watson, L., Pearce, K., Foster, J. T., et al., 2011: Multi-drug resistant Staphylococcus aureus in US meat and poultry. Clin. Inf. Dis., 53, 2, 1-4.

45. Weese, J. S., 2004: Methicillin-resistant Staphylococcus aureus in horses and horse personnel. Vet. Clinic North Am. Equine Pract., 20, 601-613.

46. Williams, N. J., Deacon, V., Pinchbeck, G., Dawson, S., 2007: A pilot survey of the prevalence of methicillin-resistant Staphylococcus aureus (MRSA) and other methicillin resistant staphylococci (MRS) nasal carriage in small animal veterinary personnel. International British Small Animal Veterinary Association Congress, Birmingham, October 2007.

47. Wulf, M. W., Sorum, M., van Nes, A., Skov, R., Melchers, W. J., Klaassen, C. H., Voss, A., 2008: Prevalence of methicillin-resistant Staphylococcus aureus among veterinarians: an international study. J. Clin. Microbiol. Inf., 14, 1, 29-34. DOI: 10.1111/j.1469-0691.2007.01873,x.

Received July 9, 2020

Accepted October 27, 2020 